\title{
CHARACTERIZATION OF A NOVEL TEMPERATURE LIDAR RECEIVER BY MEANS OF LABORATORY RAYLEIGH-BRILLOUIN SCATTERING MEASUREMENTS
}

\author{
Jiaqi Xu1 ${ }^{1,2, ~ *, ~ B e n j a m i n ~ W i t s c h a s ~}{ }^{1}$, Kun Liang ${ }^{2}$, Yuanqing Wang ${ }^{3}$, Wim Ubachs ${ }^{3}$ \\ ${ }^{1}$ German Aerospace Center (DLR), Institute of Atmospheric Physics, 82234 Oberpfaffenhofen, Germany \\ ${ }^{2}$ School of Electronic Information and Communications, Huazhong University of Science and Technology, \\ Wuhan, Hubei, 430074, China \\ ${ }^{3}$ Department of Physics and Astronomy, LaserLaB, VU University, 1081 HV Amsterdam, Netherlands \\ *Email: jiaqi.xu@dlr.de
}

\begin{abstract}
A new receiver setup based on an imaging Fizeau interferometer measuring the Rayleigh Brillouin scattering (RBS) spectra to retrieve tropospheric temperature profiles is introduced. The optical layout of the proposed receiver design is discussed and its functionality is demonstrated by means of accurate and controlled laboratory RBS measurements. The proposed temperature receiver is based on a Fizeau interferometer and a 32 channel PMT array which can resolve the RBS spectrum without applying any scanning procedures. In order to avoid any incidence angle fluctuations on the Fizeau interferometer caused by for instance atmospheric turbulence, the application of a fiver coupled telescope was investigated. It is shown that a $50 \mu \mathrm{m}$ fiber $(\mathrm{NA}=0.12)$ provides both, sufficient coupling efficiency and sufficiently low divergence in order to keep the Finesse of the interferometer. The performance of the novel lidar receiver is verified by means of accurate laboratory RBS measurements.
\end{abstract}

\section{INTRODUCTION}

Temperature is a key parameter in the atmosphere and plays a major role in many atmospheric processes. The global observation of temperature is important in predicting the atmospheric state, as well as retrievals of other atmospheric properties such as wind, pressure or other trace gas concentrations.

Currently, various in-situ and remote sensing technologies are available to obtain global temperature data. To get temperature profiles with both, high horizontal and vertical resolution and high accuracy, it would be beneficial to utilize the lidar technology. For instance, Raman lidar can be used to acquire temperature profiles with high vertical and temporal resolutions ${ }^{[1-3]}$. and are discussed to be designable for airborne ${ }^{[4]}$ and space-borne application ${ }^{[5]}$. However, due to the low Raman differential backscattering cross section, high-power lasers and large telescopes would be needed for this purpose

As the differential backscattering cross section is more than a factor of 50 larger than the one of rotational Raman scattering, it is beneficial to use Rayleigh scattering in order to derive temperature [6-8]. In particular, atmospheric temperature can be derived by resolving the RBS spectrum with high spectral resolution filters, such as atomic vapor cells ${ }^{[8]}$ or Fabry-Pérot interferometers ${ }^{[6]}$, and subsequently relating the measured spectrum to an appropriate line shape model. Hua et al. introduced a Rayleigh-Mie lidar for daytimetemperature profiling of the troposphere using a frequency tripled Nd: YAG laser $(\lambda=355 \mathrm{~nm})$ and, for the first time, a Fabry Perot interferometer (FPI) ${ }^{[6]}$. They showed temperature profiles which fit well to radiosonde temperatures from ground to $4 \mathrm{~km}$ and calculated a temperature uncertainty of less than $1 \mathrm{~K}$ within this range. Witschas et al based on a Doppler wind lidar system to obtain the RBS spectrum. In order to do so, the frequency of the used laser was changed in defined steps over a range of $12 \mathrm{GHz}$ (at 354.89 $\mathrm{nm}$ ) and a Fabry-Perot interferometer was used as frequency discriminator. The sampling of the entire RBS line shape required $28 \mathrm{~min}$, and the results show temperature profiles from $2 \mathrm{~km}$ to $15.3 \mathrm{~km}^{[9]}$.

To further reduce the measurement time and stabilize the measurement procedure, it is suggested to sample the RBS spectrum without applying any scanning procedures. This can be realized by means of an imaging Fizeau interferometer and a multichannel photomultiplier 
(PMT) array. By using such a setup, even a varying aerosol load would not negatively influence the temperature retrieval.

\section{The improved new receiver system}

The suggested temperature receiver optical design is sketched in Fig. 1.

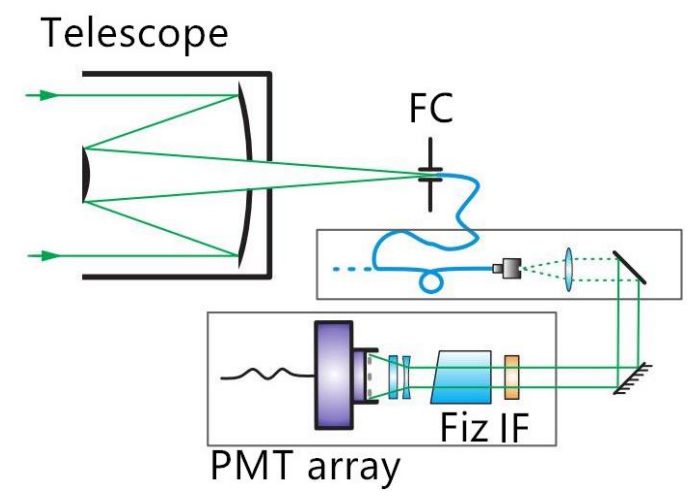

Fig. 1. Setup principle of an imaging high spectral resolution temperature receiver by using a Fizeau interferometer.

The specifications of the suggested temperature lidar receiver are listed in Table 1.

Table 1. Parameters of the Fizeau interferometer

\begin{tabular}{ll}
\hline Interferometer & Fizeau \\
Type & 19 \\
Nominal plate distance $/(\mathrm{mm})$ & 8 \\
$\mathrm{FSR} /(\mathrm{GHz})$ & 8.3 \\
Wedge angle/ $(\mu \mathrm{rad})$ & 0.80 \\
Plate reflectivity &
\end{tabular}

\begin{tabular}{ll}
\hline Detector & \\
Type & 32-channel \\
& PMT
\end{tabular}

The backscattered light from the atmosphere is collected by a telescope. A fiber coupler (Fig. 1, FC) is used to couple the backscattered light and direct it to the Fizeau interferometer. By using the fiber, any incidence angle fluctuations in the atmosphere are not transported to the Fizeau interferometer and thus, do not lead to any systematic error. After the fiber coupler, the beam diameter is adapted to match the aperture size (32 $\mathrm{mm}$ ) of the Fizeau interferometer (Fig. 1, Fiz), then laser beam is sent to a narrowband interference filter (Fig. 1, IF) (bandwidth is 0.72 $\mathrm{nm}$ ) to additionally remove background light and signal from Raman scattering. Finally, the RBS spectrum or rather the convolution of the RBS spectrum with the Fizeau transmission function is adapted by two cylindrical lenses in order to match the detector size of $1 \mathrm{~mm}$ x $32 \mathrm{~mm} \mathrm{(32}$ channel PMT array). The PMT device is able to detect the very weak backscatter signal. With this setup. entire RBS spectrum can be detected at once without applying any scanning procedures.

\subsection{Characterization of the receiver system by means of laboratory measurements}

The basic idea of the suggested temperature receiver is to retrieve temperature from measured RBS spectra. This is possible as the RBS line shape depends among other quantities also on temperature. Basically, the RBS spectrum is an image of the velocity distribution of the molecules in the scattering medium, and therefore, it contains information on gas transport properties like heat capacity, thermal conductivity, shear and bulk viscosity as well as on temperature, density (pressure) and bulk velocity ${ }^{[13-15]}$. Laboratory measurements give one the possibility to perform the RBS measurements under controlled conditions regarding the aforementioned quantities.

\section{Laboratory measurements for measuring RBS spectra with the lidar receiver}

In order to investigate the feasibility of the designed receiving system, the controlled laboratory measurements are currently performed at the LaserLaB of VU University Amsterdam. A block diagram of the Laboratory experimental setup used to measure RBS spectrum in air is shown in Fig. 2.

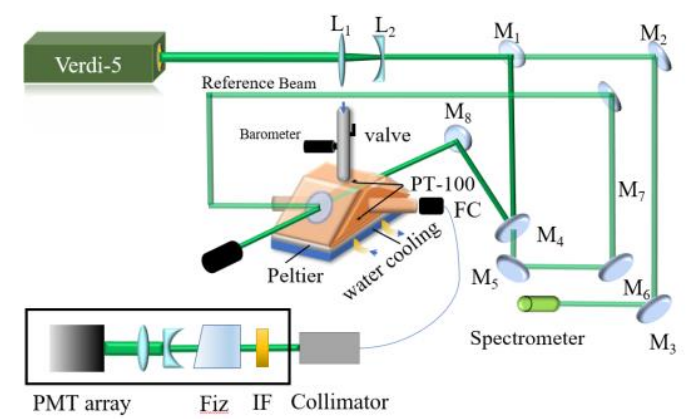

Fig. 2. Schematic diagram of the experimental setup. Dark green: laser beam $(532 \mathrm{~nm})$, light green: reference beam $(532 \mathrm{~nm}), \mathrm{SC}$ : scattering cell with Peltier and 
water-cooling to heat \& cool, Barometer: monitor the pressure, PT-100: monitor the temperature, FC: fiber coupling, Collimator: collimating laser beam, IF: narrowband filter, Fiz: Fizeau interferometer.

A Verdi-V5 laser provides continuous wave light at $532.22 \mathrm{~nm}$ at a power of 5 Watt and bandwidth less than $5 \mathrm{MHz}$. The laser light is split into two beams: The pump beam crosses the RBscattering gas cell whereas the temperature and pressure are controled and measured with an accuracy of $0.15 \%$ (pressure) and $\pm 0.2 \mathrm{~K}$ (temperature). The light scattered with a angle of $\theta=124.2^{\circ}$ is collected an directed to an optical fiber. The small fraction reference beam transmitted through M4 is used to align the beam path after the gas cell towards the detector. The scattered light is coupled to a fiber then after the narrowband interference filter (bandwidth is 0.72 $\mathrm{nm}$ ) (Fig. 2, IF) to remove background light and signal from Raman scattering, then it is analyzed the Fizeau Interferometer (Fig.2 Fiz and directed to the PMT array.

During the measurements, we found that the coupling efficiency of single mode fiber is too low for receiving scattered light, and thus, a multimode (MM) fiber was applied. However, MM fibers suffer from Speckle and reduce the Finesse of the used interferometer. Thus, investigations were performed in order to find the best compromise between light throughout and divergence. In particular we used the following fibers: $50 \mu \mathrm{m} 0.12 \mathrm{NA}$ fiber (a), $105 \mu \mathrm{m} 0.12 \mathrm{NA}$ fiber (b), $50 \mu \mathrm{m} 0.22 \mathrm{NA}$ fiber (c). First, we use the reference beam and the power meter to measure the coupling efficiency. It shows that (a) is $18.7 \%$, (b) is $28.1 \%$, (c) is $84.3 \%$.

The number of transmitted modes is also an important parameter for MM fiber selection, which will affect the finesse of the interferometer and the quality spectrum effectively obtained by The PMT. The number of modes can be calculated according to:

$$
M \approx \frac{V^{2}}{2}=\frac{\left(\frac{2 \pi \alpha}{\lambda} \cdot N A\right)^{2}}{2}
$$

where $V$ is the normalized frequency ( $V$-number), $\alpha$ is the fiber core radius, and $\lambda$ is the free space light wavelength. The mode number of fiber (a), (b), (c) is $2510,11072,8438$, respectively. The finesse of Fizeau Interferometer is determined by the ratio of FSR and the FWHM shown from different fibers, and is correlated with the fiber mode. Therefore, less modes of fiber will lead to the higher finesse of the interferometer. Secondly, less modes will result in a lower number of speckles pattern, and thus to a better image of the RBS spectrum, Considering that, the $50 \mu \mathrm{m} 0.12$ NA fiber has the lowest in the mode number, which is the best choice for our experiment,

Another important point to consider for using the fiber to collected scattering light and inject it into the interferometer is the area of the source as seen by the interferometer, which determines the étendue of the Fizeau Interferometer ${ }^{[16]}$. It must be sufficiently large to achieve the light to make the required measurements. Therefore, in order to have enough area of light source and a good collimation, we choose the Thorlabs Large-Beam Fiber Collimators (C40FC-A). By measuring the spot diameter of the fiber after the collimator, it is found that $0.12 \mathrm{NA}$ multi-mode fibers have the diameter $9.6 \mathrm{~mm}$ which is matched to the aperture size of the Fizeau interferometer.

After the optical fiber is determined, first we use a broadband LED through to measure the illumination function of the system shown in Fig. 3. After that, we can obtain the instrument function of the system using reference beam shown in Fig.4.

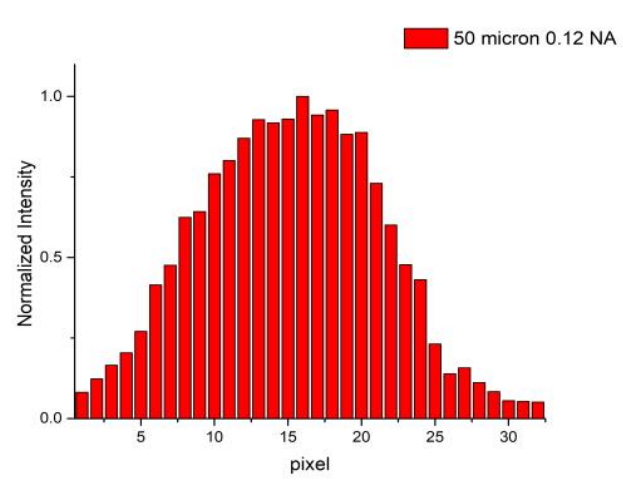

Fig. 3. The illumination function of system using the uniformly circular aperture from $50 \mu \mathrm{m} 0.12$ NA. 


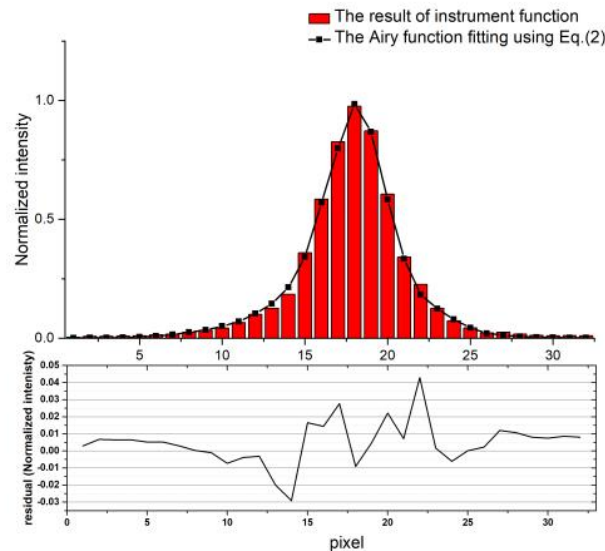

Fig. 4. top: The instrument function measured with the PMT array (red bar) and the best fitting using Eq. (2) of an Airy function (black dots), bottom: the residual of the fitting.

The transmission function of an Fizeau Interferometer can be described by an Airy function $\operatorname{Airy}(f)$ because of the lower reflectivity and lower asymmetry. Then the instrument function $I(f)$ shown in Fig. 4. can be fitted by the Airy function multiply by illumination curve:

$$
\begin{aligned}
I(f) & =\operatorname{ill}(f) * \operatorname{Airy}(f) \\
& =\operatorname{ill}(f) * \frac{1}{F S R}\left(1+2 \sum_{k=1}^{\infty} R^{k} \cos \left(\frac{2 k \pi f}{F S R}\right)\right)
\end{aligned}
$$

$I(f)$ is the instrument function of system, $\operatorname{ill}(f)$ is the illumination curve from PMT array, and the $\operatorname{airy}(f)$ is the airy function which can be calculated by the Free spectrum range (FSR) of interferometer and reflectivity $(R)$. From the fitting results, the FWHM calculate from FSR and $\mathrm{R}$ of our Fizeau interferometer system is 4.66 pixel, The FSR (30.76 pixel) and the FWHM of the fringe give a finesse of 6.60 .

In order to verify the feasibility of the receiving system to obtain the scattered light, we will first measure the gas $\mathrm{CO} 2$ which has a high scattering cross section. The averaging spectrum shown in Fig.5 is sum of 10000 shots from PMT, which only need for $100 \mathrm{~s}$ (one shot is $10 \mathrm{~ms}$ ), and average for a more accurate experiment results and removing the influence of random noise.

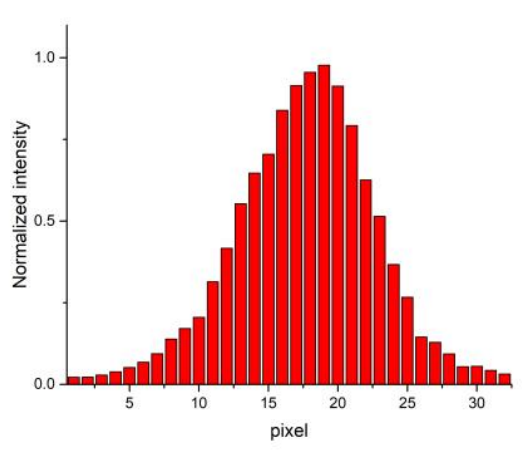

Fig. 5. The RBS spectrum of $\mathrm{CO} 2$ at pressure 3.00 bar, temperature $293.0 \mathrm{~K}$.

Further work is to acquire the RBS spectrum of air from different and the pressure with the new receiver system in the lab, then the explained retrieval algorithms (both analytical model and Tenti S6 model) are applied to the measured RBS line shapes. Latest results of this retrieval will be discussed during the conference.

\section{ACKNOWLEDGEMENTS}

Jiaqi $\mathrm{Xu}$ is supported by the China Scholarship Council (CSC).

\section{REFERENCES}

[1] A. Behrendt, et al. Appl. Opt. 43, 2930 (2004)

[2] M. Radlach, et al. Atmos. Chem. Phys. 8, 159 (2008)

[3] A. Behrendt et al. Appl. Opt. 39, 1372 (2000)

[4] M. Fraczek, et al. Appl. Opt. 51, 148 (2012)

[5] P. Di Girolamo, et al. Appl. Opt. 45, 2474 (2006)

[6] Hua D., et al. Appl. Opt., 44(7): 1315-1322 (2005)

[7] Hair, J., et al. Appl. Opt., 40(30): 5280-5294 (2001)

[8] Liu, et al. Appl. Opt., 48(27): 5143-5154 (2009)

[9] B. Witschas, et al. The 27th International Laser Radar Conference (ILRC 27). EDP Sciences 119, 27004 (2016)

[10] B. Witschas, in Atmospheric Physics: Background Methods Trends, U. Schumann, ed. (Springer), 69-83

[11] Paffrath, U. PhD thesis, Technical university of Munich. (2006)

[12] O. Reitebuch, et al, J. Atmos. Ocean. Technol. 26, 2501 (2009)

[13] B. Witschas, et al, Appl. Opt. 51, 6207 (2012)

[14] B. Witschas, et al. Opt. Lett. 39(7): 1972-1975 (2014)

[15] K. Liang, et al. sensors, 17, 1503 (2017)

[16] O. Novák, et al. Review of sci. instrument 82 , 023105 (2011) 